\title{
The influence of advertisements on the conspicuity of routing information
}

\author{
Theo Boersema* and Harm J.G. Zwaga ${ }^{\dagger}$ \\ *Delft University of Technology; ${ }^{\dagger}$ University of Utrecht
}

\begin{abstract}
An experiment is described in which the influence of advertisements on the conspicuity of routing information was investigated. Stimulus material consisted of colour slides of 12 railway station scenes. In two of these scenes, number and size of advertisements were systematically varied. Subjects were instructed to locate routing signs in the scenes. Performance on the location task was used as a measure of the routing sign conspicuity. The results show that inserting an advertisement lessens the conspicuity of the routing information. This effect becomes stronger if more or larger advertisements are added.
\end{abstract}

\section{Keywords: Route indicators, advertising signs, environment}

\section{Introduction}

It may be assumed that the presence of advertisements in public buildings (airport terminals, railway stations, shopping centres) diminishes the ability of people to locate routing information. In more general terms, the conspicuity of the routing information is expected to be low when advertisements are present in the environment where it is displayed.

The visual conspicuity of an object presented at an unknown location in a stimulus field can be operationally defined in terms of the probability that the object will be noticed by an observer within a given period of time or in terms of the time needed to locate the object with a certain probability. The visual conspicuity of an object is determined by:

- the physical properties of the object itself,

- the relation between these physical properties and those of the complete stimulus field, i e, the 'contrast' between the object and its environment,

- cognitive factors.

With regard to the physical properties of objects and environments, it has been shown that differences in size, colour, shape, location, luminance and number influence the conspicuity of objects (Eriksen, 1953; Engel, 1974; Jenkins and Cole, 1982; Cole and Jenkins, 1984). Apart from these static properties, dynamic physical properties, like luminance change and relative movement, can also affect conspicuity (Crawford, 1963; Lewis, 1975).

The effect of cognitive factors on conspicuity has also been demonstrated. Biederman et al (1973) showed that the detection rate of targets was lower for jumbled versions of real-world scenes than for natural versions of the scenes. This suggests the influence of the observers' pre-knowledge of the global structure of the scenes. Antes (1977) reported that both recognition and localisation accuracy of sections of real-world scenes depended on their rated informativeness. Loftus and Mackworth (1978) showed that objects with a low probability of appearing in a certain environment are fixated earlier, more often, and with longer durations than objects having a high appearance probability. In contrast with the relatively stable cognitive properties such as experience and knowledge, the effect of which has been illustrated in the examples above, man also has capabilities for momentary adjustments of effort to the aspired level of task performance, as pointed out by Kahneman (1973). He takes these adjustments together under the term set. Experimental evidence in the field of conspicuity for such a temporary and task dependent adjustment has been reported by Jonides and Gleitman (1972). They showed that the conspicuity of a target depends on the conceptual categorisation of the target and the other objects in the stimulus field: in a stimulus field of digits, the stimulus " 0 " was more conspicuous when called the letter $O$ than when called the digit zero. This experiment was replicated by Butler (1980) with the same results, but in a replication by Duncan (1983) the phenomenon failed to appear. Green and Anderson (1956), Smith (1962) and Williams (1966) showed that information given in the instruction about the physical properties of a target, like colour and size, is very effective in increasing search efficiency. In our pilot experiments for this study we were able to show that manipulating set by giving different instructions (searching for a specific type of target in real-world scenes or just 
reporting the objects which had been noticed) strongly influenced the performance. In this context, Cole and Hughes (1984) make a distinction between attention conspicuity and search conspicuity. They define attention conspicuity as the capacity of an object to attract the attention of an observer who is not instructed to search for a target nor even told that one of the objects presented is the target. Search conspicuity is described by them as the property of an object that enables it to be quickly and reliably located by an observer searching for it. They report on a field trial in which both kinds of conspicuity were measured. One of their results was that the number of target hits for search conspicuity was about three times that of attention conspicuity. This result clearly demonstrates the effect of the set of the observer, manipulated through the instruction, on the conspicuity of an object.

The aim of this study is to determine, given a specific set of the subject (a search task), to what extent the physical properties of the environment of a target have effect on its visual conspicuity. The stimulus fields show real-world scenes with the routing signs as targets in the search task. By adding to the scenes advertisements differing in number and size, the physical properties of the vicinity of the targets are varied. The conspicuity of the routing signs is measured in terms of search task performance. It is expected that the conspicuity of the routing signs will decrease with increasing number and size of the advertisements.

\section{Method}

\section{Subjects}

Subjects were 156 undergraduate students (male and female) with at least normal or corrected-to-normal vision. They received course credit or were paid for their participation.

\section{Stimulus material}

Stimuli consisted of colour slides showing 12 scenes in railway stations, containing routing signs in many different shapes and sizes. However, on all routing signs the information was presented in white on a blue background. In two of these scenes, number and size of the advertisements were systematically varied using photographic techniques. From each of the two scenes seven types of experimental slides were obtained: one type showing the scene with routing information only (type 0 ), three types with one, two and three small advertisements added respectively $(1 S, 2 S$, $3 \mathrm{~S}$ ), and three types with large advertisements added (1L, $2 \mathrm{~L}, 3 \mathrm{~L}$ ). The area of a small advertisement was twice the area of a routing sign, and a large one occupied four times that area. One of the experimental scenes (scene A) contained only one routing sign. The size of this sign was used as a reference for the size of the area of the advertisements. The advertisements, which never contained the colour blue of the routing signs, were inserted directly above the routing sign. Examples of the experimental slides of scene A are shown in Fig. 1. The other experimental scene (scene B) contained a (large) reference sign and additionally three smaller signs: one to the right and two to the left of the reference sign. Scene B is illustrated in Fig. 2. The exact content of the remaining scenes is not described here as only the relative effects of number and size of advertisements in the seven types of the experimental scenes were studied.
Apparatus

Two Kodak Carousel S-AV2030 slide projectors were used: one to project the scene slides, the other to project a grid slide. The distance between subject and screen was $210 \mathrm{~cm}$. The projected picture size was $71.0 \times 47.5 \mathrm{~cm}$, thus subtending a horizontal visual angle of about 20 degrees. The horizontal visual angle of the reference sign was 43 minutes in scene $A$ and 1 degree 8 minutes in scene $B$

\section{Procedure and experimental design}

There were seven experimental conditions: one for each of the seven types of experimental slides. In each condition a subject was shown 12 slides: two experimental slides (one of scene A and one of scene B) of the same type and ten slides showing the other scenes. Each subject was shown the slides in one of four orders. These orders were random with one restriction: the experimental slides never appeared in one of the first three positions. The exposure time was $1 \mathrm{~s}$. The subjects were instructed to tell where they had seen white on blue routing information in the scene using as an aid a $4 \times 6$ grid of numbered squares projected after each scene at the same location as the slide of the scene. A routing sign was scored as correctly located if the subject named its correct or an adjacent grid square. Only the responses on the experimental slides were used for further analysis. Subjects were randomly assigned to one of seven independent groups Group size was 36 for condition 0 and 20 for the other six conditions.

\section{Results}

Results and statistical analyses are presented separately for each of the two experimental scenes because of the undefined qualitative differences between the two scenes.

\section{Scene A}

Table 1 summarises the results for scene $A$. The data were subjected to a two-way analysis of variance to determine the effects of number $(1,2,3)$ and size (small, large) of the advertisements. The data for condition 0 were excluded from this analysis because they are not related to the factor size. The analysis (Table 2) shows that the effect of size is significant at $p<0.01$. The number and the interaction effects are not significant.

This latter result permits the combination of the data for the small and large advertisements (Table 3 ) and to perform a one-way analysis of variance on the effect of number $(0,1,2,3)$. This analysis indicates that the effect is significant $(F(3,152)=7.438, p<0.001)$. Subsequently, the differences between all pairs of treatment means were subjected to an a posteriori test according to Scheffé's method. This analysis shows that condition 0 differs significantly from each of the other conditions (p's $<0 \cdot 10)$; none of the other differences is significant (p's $>0 \cdot 10$ ).

\section{Scene B}

Scoring for scene B differed from that used for scene A. Subjects correctly locating one or two routing signs in the centre as well as in the right-hand part of the scene received a score of 1 . The score for subjects locating either one or two 


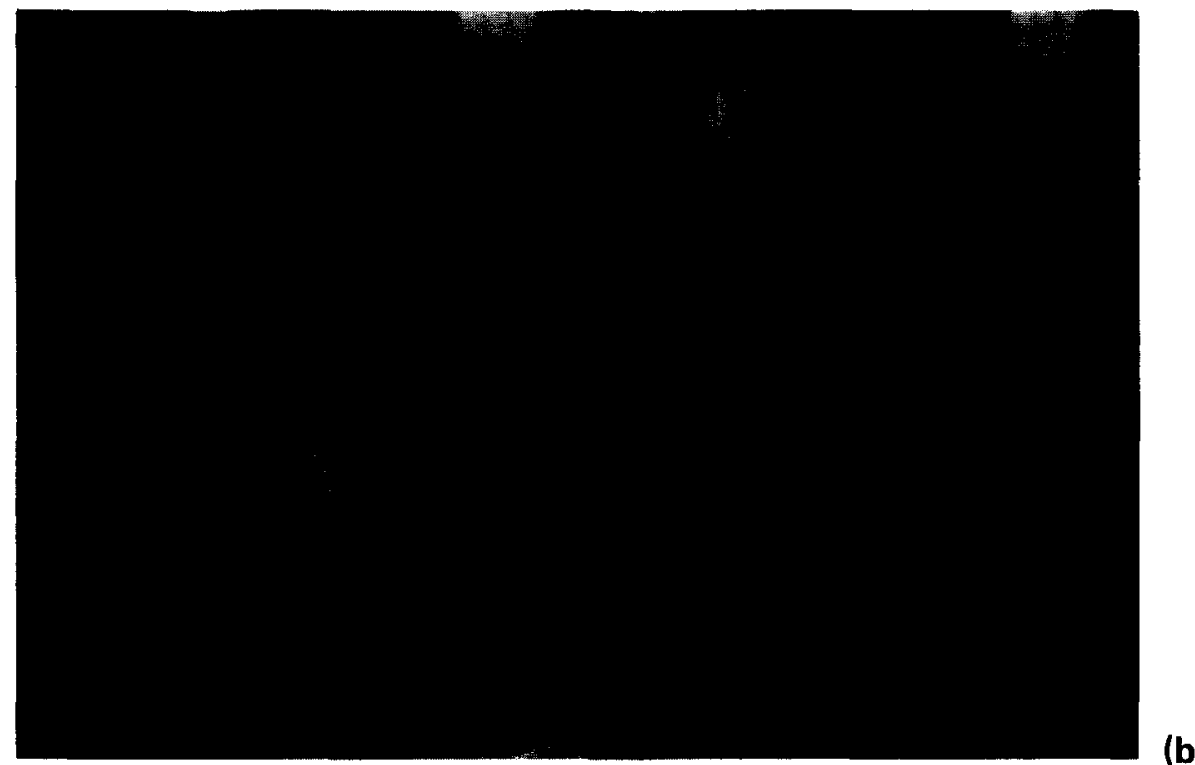

Fig. 1 Scene A with the route sign (blue with white lettering) under the left-hand arch, shown in (a) with no advertisements, (b) with one small yellow advertisement, and (c) with three large advertisements (green, yellow and red). Due to the production technique, the colour slides used as stimulus material and shown in Figs. 1 and 2 were rather 'grainy' This did not hamper the subjects who saw the slides only during $1 \mathrm{~s}$.

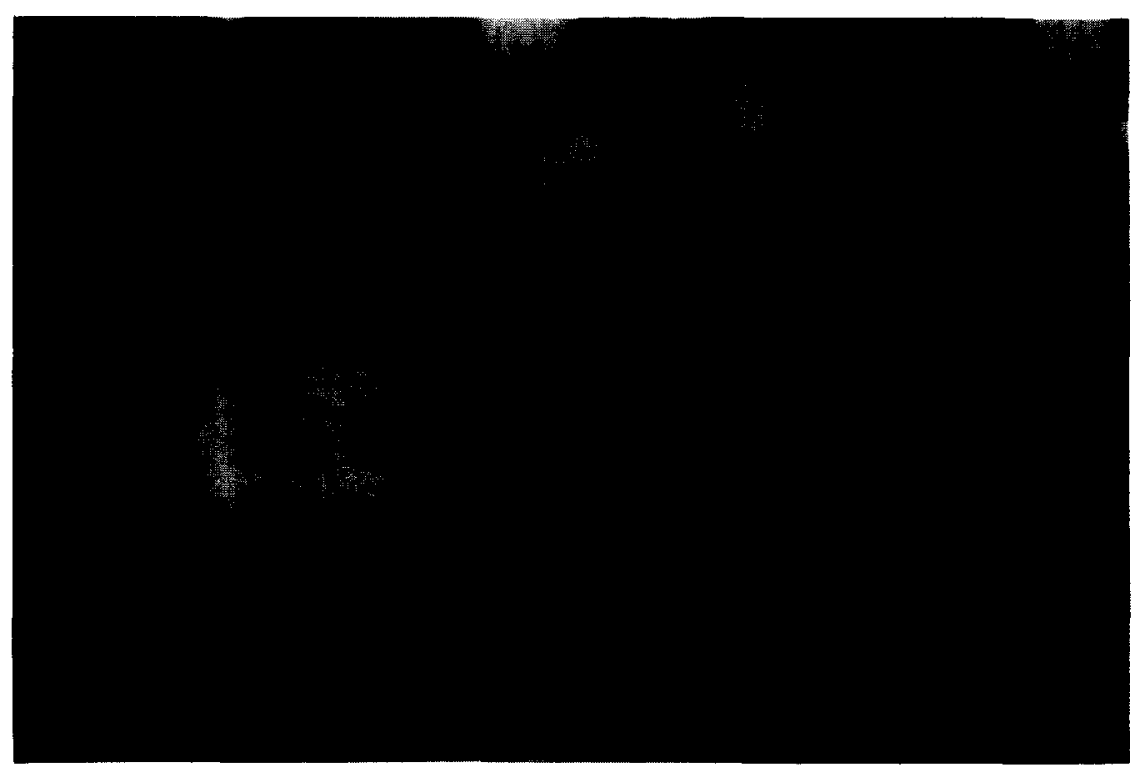




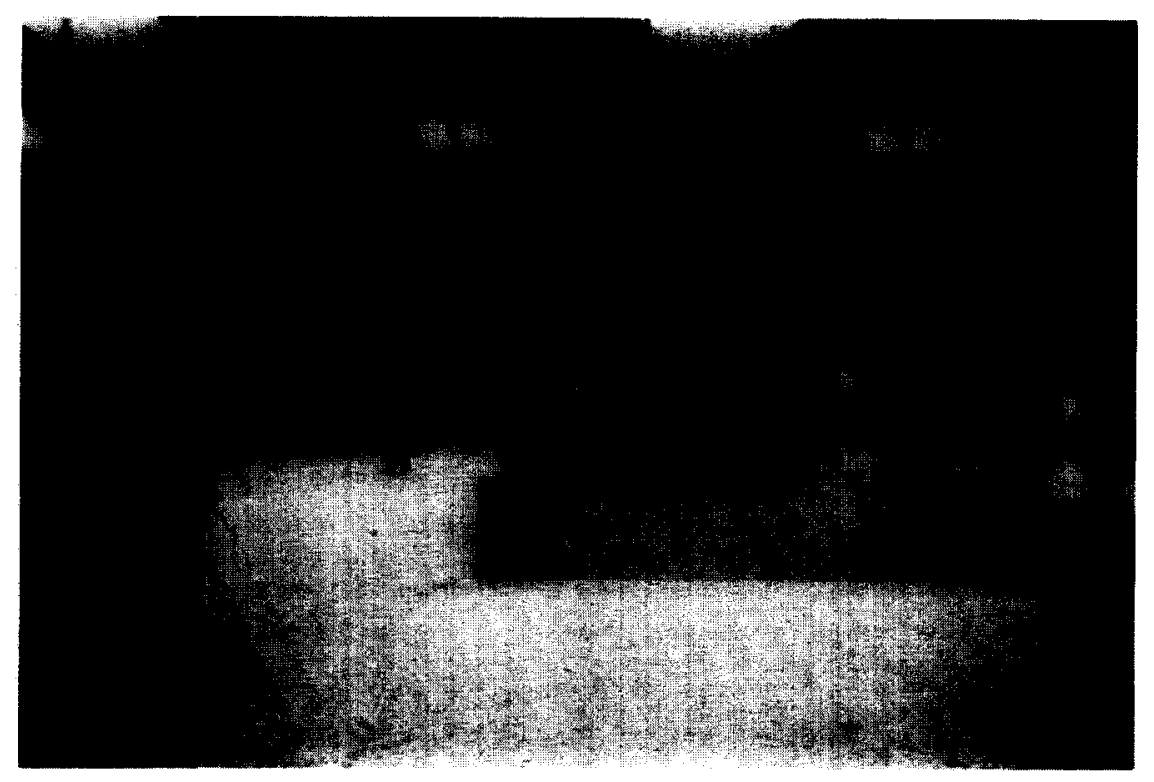

(a)

Fig. 2 Scene B with the routing sign to the right of the centre kiosk, with three small advertisements (a) and with two large advertisements (b). (b)

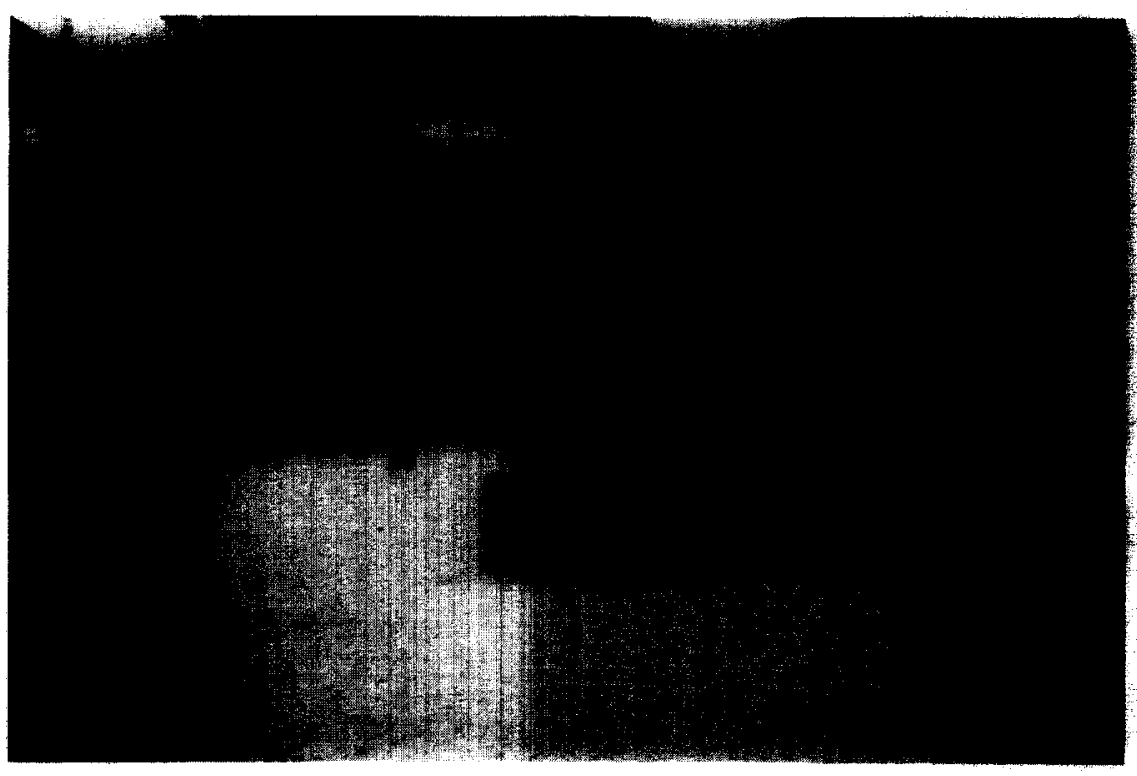

routing signs in the centre or one or two on the right was 0.5 . The results are summarised in Table 4. For scene B the same statistical analyses were performed as for scene $A$. The two-way analysis (Table 5) shows significant effects of number and size and no significant interaction effect. Further analysis, with the data aggregated for small and large advertisements (Table 6), demonstrates that the effect of number $(0,1,2,3)$ is significant $(F(3,152)=13 \cdot 744$, $\mathrm{p}<0.001)$. The multiple comparison of the treatment means shows only significant differences between conditions 0 and 1,0 and 2 and 0 and 3 (p's $<0.01$ ).

Table 7 presents a summary of the subjects' locating performance for the routing signs in the centre and in the right-hand part of scene B. In Table 8, derived from Table 7, the first row gives the percentages of subjects having seen at least routing signs in the centre of the scene (first and second row of Table 7). The percentages of subjects having located at least the routing signs in the right-hand part of the scene are presented in the second row (first and third row of Table 7). Although no predictions were formulated about the effect of the different positions of routing signs in the scene nor about the effect of their positions in relation to the advertisements, the data in Table 8 seem to allow the following comments. First, in the condition without advertisements, subjects more often notice the routing signs located in the centre than those located on the right $(72 \cdot 3$ against $47 \cdot 3 \%$ ). Second, inserting advertisements causes, independently of their size and number, a substantial decrease in the number of subjects locating at least routing signs in the centre of the scene. Third, considering only subjects locating at least routing signs in the right-hand part of the scene, there is a difference between the effects of inserting small and large advertisements in the vicinity of those signs: the decrement caused by small advertisements is negligible whereas that of large advertisements is considerable.

\section{Discussion}

The results indicate for both experimental scenes a significant decrement of the conspicuity of routing signs if an advertisement is inserted in their vicinity. This appears to be the case despite the fact that the colours of the signs are 
Table 1: Routing sign locating performance in scene $A$. The first row shows the percentage of subjects correctly locating the routing sign (score 1). The second row shows the percentages of subjects who missed the sign (score 0 ). The bottom row shows the mean score per condition (expressed as a probability of success).

\begin{tabular}{cccccccc}
\hline & \multicolumn{8}{c}{ Condition } \\
\cline { 2 - 8 } Score & 0 & $1 S$ & $2 S$ & $3 S$ & $1 L$ & $2 L$ & $3 L$ \\
\hline 1 & 66.7 & 50.0 & 40.0 & 30.0 & 30.0 & 15.0 & 10.0 \\
0 & 33.3 & 50.0 & 60.0 & 70.0 & 70.0 & 85.0 & 90.0
\end{tabular}

$\begin{array}{llllllll}\text { mean } & 0.667 & 0.500 & 0.400 & 0.300 & 0.300 & 0.150 & 0.100\end{array}$

Table 2: Scene A. Summary of the two-way analysis of variance on the effects of number and size of advertisements.

\begin{tabular}{lrccc}
\hline Source & df & MS & $F$ & $P$ \\
\hline number & 2 & 0.408 & 2.064 & 0.132 \\
size & 1 & 1.408 & 7.120 & 0.009 \\
number x size & 2 & 0.008 & 0.042 & 0.959 \\
residual & 114 & 0.198 & & \\
\hline
\end{tabular}

Table 3: Scene A. Data added over the conditions with small and large advertisements. The entries are based on the percentages given in Table 1.

\begin{tabular}{ccccc}
\hline & \multicolumn{4}{c}{ Condition } \\
\cline { 2 - 5 } Score & 0 & 1 & 2 & 3 \\
\hline 1 & 66.7 & 40.0 & 27.5 & 20.0 \\
0 & 33.3 & 60.0 & 72.5 & 80.0 \\
\hline mean & 0.667 & 0.400 & 0.275 & 0.200 \\
\hline
\end{tabular}

known to the observers and despite their fair notion of the areas in the scenes where the signs can be found. For the observers, the more uncertain properties of the signs are their exact shape and size. A possible explanation for the effect of one advertisement on locating routing signs is the correspondence in shape and position between advertisements and routing signs. This correspondence forces the subjects to process these similar elements up to a certain degree before an advertisement can be classified as a non-target. Apparently, the induced set of the subjects is not strong enough to let them ignore the advertisements completely. The presence of an advertisement appears to lessen the 'contrast' between a routing sign and the remainder of the scene. Or, in the terms of Cole and Hughes (1984), the attention conspicuity of the advertisement is so much larger than that of the corresponding area in condition 0 that the search conspicuity of routing signs suffers significantly.

For both scenes, though statistically not significant in the case of scene $A$, the conspicuity of the routing signs is further diminished as the number of advertisements increases. This effect is stronger for the larger advertisements than for the smaller ones. From these results it can be concluded that number and size of advertisements in a scene are relevant physical properties with regard to the conspicuity of routing signs. A simple linear model based on area occupied by advertisements does not fully explain the combined effects of their number and size. With equal total areas, the effect of two small advertisements is less than the effect of one large advertisement. Also, the effect of three small advertisements is smaller than the average effect of one and two large advertisements. A model to predict the effect of number and size of advertisements or their combined effect appears difficult to formulate with the results of only two scenes available.

The results for scene B allow some tentative conclusions as to the effects of the relative positions of routing signs and advertisements in a scene. First, if no advertisements are present in the scene, the signs in the centre are noticed more often than those on the right though one of these signs is much larger than the other ones. It seems that subjects start their one second search in the centre of the scene. Second, when advertisements are inserted in the scene, the percentage of subjects noticing at least routing signs in the centre decreases considerably. The presence of advertisements

Table 4: Routing sign locating performance in scene $B$. The first row shows the percentages of subjects locating one or two routing signs in the centre as well as one or two in the right-hand part (score 1). The second row shows the percentages of subjects noticing one or two routing signs either in the centre or in the right-hand part (score 0.5 ). The third row shows the percentages of subjects who missed all routing signs (score 0 ). In the bottom row the mean score per condition is given.

\begin{tabular}{lccrrrrr}
\hline & \multicolumn{7}{c}{ Condition } \\
\cline { 2 - 8 } Score & 0 & $1 S$ & $2 S$ & $3 S$ & $1 L$ & $2 L$ & $3 L$ \\
\hline 1 & 41.7 & 20.0 & 5.0 & 5.0 & 0.0 & 5.0 & 5.0 \\
0.5 & 36.1 & 40.0 & 65.0 & 25.0 & 40.0 & 25.0 & 5.0 \\
0 & 22.2 & 40.0 & 30.0 & 70.0 & 60.0 & 70.0 & 90.0 \\
\hline mean & 0.597 & 0.400 & 0.375 & 0.175 & 0.200 & 0.175 & 0.075 \\
\hline
\end{tabular}


Table 5: Scene B. Summary of the two-way analysis of variance on the effects of number and size of advertisements.

\begin{tabular}{lrccc}
\hline Source & df & MS & F & P \\
\hline number & 2 & 0.358 & 4.147 & 0.018 \\
size & 1 & 0.833 & 9.645 & 0.002 \\
number x size & 2 & 0.033 & 0.386 & 0.681 \\
residual & 114 & 0.086 & & \\
\hline
\end{tabular}

Table 6: Scene B. Data added over the conditions with small and large advertisements. The entries are based on the percentages given in Table 4.

\begin{tabular}{lcccc}
\hline \multicolumn{5}{c}{ Condition } \\
\cline { 2 - 5 } Score & 0 & 1 & 2 & 3 \\
\hline 1 & 41.7 & 10.0 & 5.0 & 5.0 \\
0.5 & 36.1 & 40.0 & 45.0 & 15.0 \\
0 & 22.2 & 50.0 & 50.0 & 80.0 \\
\hline mean & 0.597 & 0.300 & 0.275 & 0.125 \\
\hline
\end{tabular}

Table 7: Locating routing signs in scene B. Percentages of subjects locating one or two routing signs in the centre and in the right-hand part of the scene (first row), in the centre only (second row), in the righthand part only (third row) or none (fourth row).

\begin{tabular}{lrrrrrrr}
\hline & \multicolumn{8}{c}{ Condition } \\
\cline { 2 - 8 } Performance & 0 & $1 S$ & $2 S$ & $3 S$ & $1 L$ & $2 L$ & $3 \mathrm{~L}$ \\
\hline centre and right & 41.7 & 20.0 & 5.0 & 5.0 & 0.0 & 5.0 & 5.0 \\
centre & 30.6 & 5.0 & 30.0 & 0.0 & 25.0 & 20.0 & 0.0 \\
right & 5.6 & 35.0 & 35.0 & 25.0 & 15.0 & 5.0 & 5.0 \\
0 & 22.2 & 40.0 & 30.0 & 70.0 & 60.0 & 70.0 & 90.0 \\
\hline
\end{tabular}

Table 8: Locating route signs in scene $\mathrm{B}$. This table is derived from Table 7. Percentages in the first row are obtained by adding the first and second row of Table 7. They represent the subjects having seen signs in the centre and in the right or in the centre of the scene. The second row of this table is obtained by adding the first and third rows of Table 7.

\begin{tabular}{lccccccc}
\hline & \multicolumn{8}{c}{ Condition } \\
\cline { 2 - 7 } Performance & 0 & $1 S$ & $2 S$ & $3 S$ & $1 L$ & $2 L$ & $3 L$ \\
\hline$(c+r)+c$ & 72.3 & 25.0 & 35.0 & 5.0 & 25.0 & 25.0 & 5.0 \\
$(c+r)+r$ & 47.3 & 55.0 & 40.0 & 30.0 & 15.0 & 10.0 & 10.0
\end{tabular}

seems to direct the attention of the subjects to the right of the scene. Third, for subjects seeing at least a routing sign on the right, the large advertisements appear to be more distracting than the small advertisements.

The high degree of resemblance between the results of the two experimental scenes suggests than an acceptable reliability of the experimental procedure can be expected. The procedure also seems to be a sensitive measuring device. It can even show the effects of relatively small changes in the scenes on the conspicuity of the routing signs. The external validity of the procedure is, of course, difficult to assess. However, the correspondence between the task of the subjects in the experiment and the assumed behaviour of people in a large building in which they try to find their way, suggests that experiments following the described procedure can be used in practical situations where the efficiency of a routing system and the revenues from advertisements have to be weighed against one another.

\section{Acknowledgements}

Brief reports of the experiment were presented at the Ergonomics Society's Conference 1984 in Exeter, UK, and at the Human Factors Society 28 th Annual Meeting in San Antonio, Texas, USA, 1984. Financial support for preparing the stimulus material was provided by Netherlands Railways. The authors are indebted to José Roelofs for conducting the experiment.

\section{References}

Antes, J.R.

1977 Memory and Cognition, 5, 155-161. Recognising and localising features in brief picture presentations.

Biederman, I., Glass, A.L., and Stacy, E.W., Jr

1973 Journal of Experimental Psychology, 97, 22-27. Searching for objects in real-world scenes.

Butler, B.E.

1980 Canadian Journal of Psychology, 34, 238-247. The category effect in visual search: identification versus localisation factors.

Cole, B.L., and Hughes, P.K.

1984 Human Factors, 26, 299-313. A field trial of attention and search conspicuity.

Cole, B.L., and Jenkins, S.E.

1984 Vision Research, 24, 261-270. The effect of variability of background elements on the conspicuity of objects.

Crawford, A.

1963 Ergonomics, 6, 287-294. The perception of light signals: the effect of mixing flashing and steady irrelevant lights.

Duncan, $\mathbf{J}$.

1983 Perception and Psychophysics, 34, 221-232. Category effect in visual search: a failure to replicate the "ohzero" phenomenon.

Engel, F.L.

1974 Vision Research, 14, 459-471; Visual conspicuity and selective background interference in eccentric vision.

Eriksen, C.W.

1953 Journal of Experimental Psychology, 45, 126-132. Object location in a complex perceptual field. 
Green, B.F., and Anderson, L.K.

1956 Journal of Experimental Psychology, 51, 19-24.

Colour coding in a visual search task.

Jenkins, S.E., and Cole, B.L.

1982 Vision Research, 22, 1241-1252, The effect of the density of background elements on the conspicuity of objects.

Jonides, J., and Gleitman, $\mathbf{H}$.

1972 Perception and Psychophysics, 12, 457-460. A conceptual category effect in visual search: $O$ as a letter or a digit.

Kahneman, D.

1973 Attention and effort. Prentice-Hall, Englewood Cliffs.
Lewis, M.S.

1975 Perceptual and Motor Skills, 41, 411-416.

Determinants of visual attention in real-world scenes.

Loftus, G.R., and Mackworth, N.H.

1978 Journal of Experimental Psychology: Human Perception and Performance, 4, 565-572. Cognitive determinants of fixation location during picture viewing.

Smith, S.L.

1962 Journal of Experimental Psychology, 64, 434-440. Colour coding and visual search.

Williams, L.G.

1966 Perception and Psychophysics, , 315-318. The effect of target specification on objects fixated during visual search.

\section{Reprints}

\section{Reprints of all articles in this journal are available in quantities of 100 or more}

\section{Reprints are essential-}

- for the company that wants to distribute impartial comment on its activities to potential customers and clients

- for the company that wants to up-date its technical staff on new techniques and new technologies

- for the company that wants to publicize its research and development work

- for the training course organizer who wants to assemble key reading material for his students

- for the university or technical college lecturer who wants to distribute the latest information on a topic under study

For full details of prices and availability of reprints, please write to

The Reprint Department

Butterworth Scientific Limited

PO Box 63 Westbury House Bury Street

Guildford Surrey GU2 5BH England 

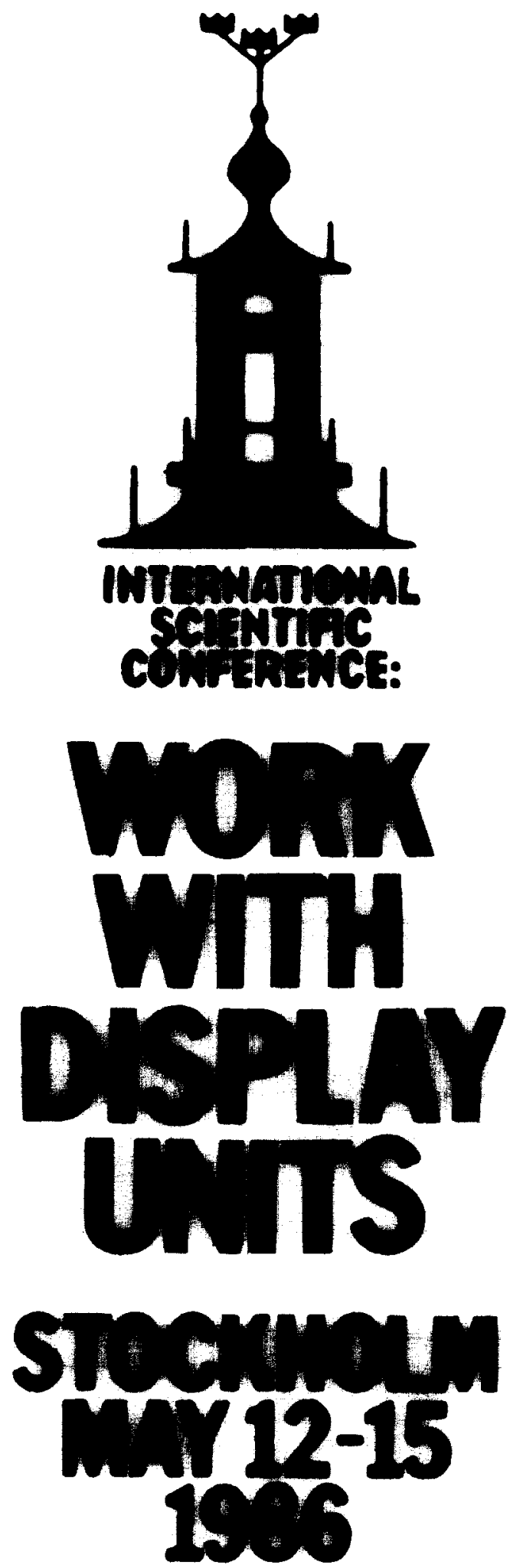

For more details please write to:

WWDU, c/o Stockholm Convention Bureau, Box 1617, S-111 86 STOCKHOLM, Sweden 